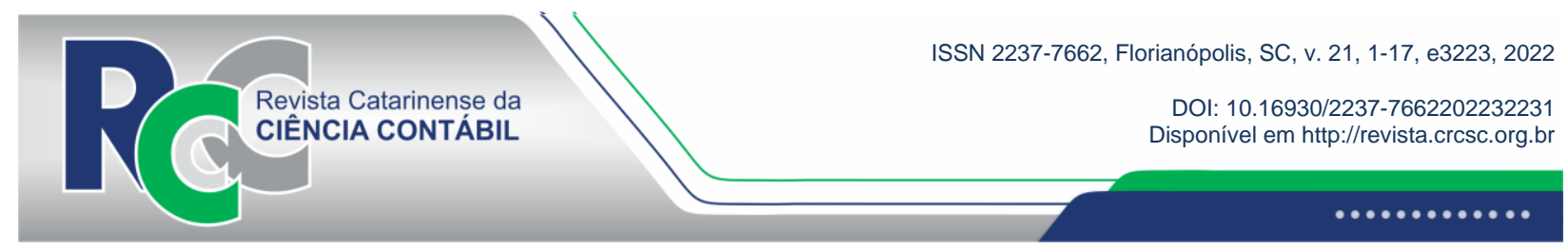

\title{
INFLUÊNCIA DA RESPONSABILIDADE SOCIAL CORPORATIVA NA QUALIDADE DAS DEMONSTRAÇÕES CONTÁBEIS DE EMPRESAS BRASILEIRAS
}

\author{
THE INFLUENCE OF CORPORATE SOCIAL RESPONSIBILITY ON THE \\ QUALITY OF ACCOUNTING STATEMENTS OF BRAZILIAN COMPANIES
}

\author{
MARIA MACILEYA AZEVEDO FREIRE \\ Centro Universitário Estácio do Ceará \\ Endereço: Avenida Duque de Caxias, 101 | Centro | 60035-111 | Fortaleza/CE | Brasil. \\ (1) https://orcid.org/0000-0002-8167-0473 \\ macileya@hotmail.com
}

\author{
ANTONIO RODRIGUES ALBUQUERQUE FILHO \\ Centro Universitário Estácio do Ceará \\ Endereço: Avenida Duque de Caxias, $101 \mid$ Centro | 60035-111 | Fortaleza/CE | Brasil. \\ (1) https://orcid.org/0000-0003-2108-3979 \\ antoniofilhoufc@hotmail.com
}

\section{RESUMO}

Diante de um mercado cada vez mais exigente, consciente das necessidades e efeitos das ações organizacionais, cresce o interesse de evidenciação e controle das informações divulgadas. Assim, com o objetivo de investigar a influência da Responsabilidade Social Corporativa (RSC) na qualidade das demonstrações contábeis de empresas brasileiras, realizou-se uma pesquisa descritiva junto a 300 empresas listadas na B3, aplicando-se estatística descritiva, teste de diferença entre médias, correlação e regressão logística para o tratamento dos dados. Foram analisadas a Responsabilidade Social Corporativa (RSC) medida pela métrica geral CSRHub, e a qualidade da informação representada pela republicação dos seus demonstrativos financeiros, considerando-se que esse parâmetro sinaliza baixa qualidade da informação inicialmente divulgada. Os resultados evidenciaram que existem diferenças estatisticamente significantes na RSC referente às empresas que republicam suas demonstrações contábeis comparadas as empresas que não republicam seus demonstrativos, denotando que, as empresas que não republicam suas informações tendem a apresentar um maior investimento em RSC. Constatou-se, ainda, que quanto maior a RSC e o tamanho organizacional, menor a republicação das demonstrações contábeis pelas empresas. Por fim, verificou-se que existe uma influência negativa da RSC na republicação das demonstrações contábeis, apresentando que quanto maior a RSC, menor a probabilidade de republicação das demonstrações contábeis pelas empresas. Desta forma, empresas com maiores níveis de RSC apresentam maior possibilidade de elevar os cuidados com a informação divulgada,

Editado em português e inglês. Versão original em português.

Recebido em 8/11/2021. Revisado em 30/12/2021. Aceito em 24/01/2022 pelos Prof. Dr. Sérgio Murilo Petri (Editor-Chefe) e Prof. Dr. Sandro Vieira Soares (Editor Adjunto). Publicado em 11/02/2022.

Copyright (C) 2022 RCCC. Todos os direitos reservados. É permitida a citação de parte de artigos sem autorização prévia, desde que identificada a fonte. 
e, assim, denotar maior qualidade da informação contábil. Dessa maneira, aceitou-se a hipótese deste estudo de que empresas que apresentam maiores práticas de RSC divulgam demonstrações contábeis de maior qualidade.

Palavras-chave: Responsabilidade Organizacional. Qualidade da informação Contábil. Republicação de demonstrações Contábeis.

\begin{abstract}
The interest in revealing and controlling disclosed information grows due to an increasingly demanding market that is aware of the need and effect of organizational actions. Thus, a descriptive survey was conducted with 300 companies listed on B3 in order to investigate the influence of Corporate Social Responsibility (CSR) on the quality of financial statements of Brazilian companies, treating data by applying descriptive statistics, test of difference between means, correlation, and logistic regression. We analyzed the Corporate Social Responsibility (CSR) measured by the general metric CSRHub, and the quality of information represented by the republishing of their financial statements, considering that this parameter signals low quality of the information initially disclosed. The results showed that there are statistically significant differences in the CSR concerning the companies that republish their financial statements compared to those that do not, denoting that their tends to be a higher investment in CSR in companies that do not republish their information. It was also found that the greater the CSR and the organizational size, the lower the republishing of the financial statements by the companies. Finally, it was found that the CSR negatively affects the republishing of the financial statements, presenting that the higher the CSR, the lower the probability of that the companies will republish the financial statements. Thus, companies with higher CSR levels are more likely to increase the care with the information disclosed, therefore denoting higher quality of accounting information. Thus, the hypothesis of this study that companies with higher CSR practices disclose higher quality financial statements was accepted.
\end{abstract}

Keywords: Organizational Responsibility. Quality of Accounting Information. Republishing Financial Statements.

\title{
1 INTRODUÇÃO
}

Diante de uma maior conscientização das necessidades e expectativas geradas em uma empresa, cresce o interesse organizacional de fatores associados e maiores exigências do mercado de capitais quanto aos cuidados e controle na evidenciação de informações (Silva, Noriller, Silva, \& Niyama, 2018). As organizações vêm buscando novas estratégias e mecanismos que representem maior segurança para os investidores e local em que estão inseridas, desta forma, que reflitam não só no desempenho da entidade, mas quanto aos impactos positivos da empresa em seu ambiente social (Berens, Van Riel, \& Van Rekom, 2007; Koprowski, Krein, Barichello, Mazzioni, \& Dal Magro, 2019).

Nessa perspectiva, a Responsabilidade Social Corporativa (RSC) apresenta-se como instrumento de grande valor organizacional, isso se deve ao fato de corresponder a uma responsabilidade mais ampla da organização para seus diversos stakeholders (Rao \& Tilt, 2016). Deste modo, inclui práticas que direcionam maiores cuidados, ligadas a proteção ambiental, relações com as comunidades locais, condições de trabalho, doações, governança corporativa (Berens et al., 2007), assim como a divulgação de informações financeiras mais transparentes e 
mais seguras para todas as partes interessadas, em comparação às organizações que não atendem aos mesmos critérios sociais (Kim, Park, \& Wier, 2012).

Nesse ínterim, a qualidade da informação é algo primordial (Silva, Takamatsu, \& Avelino, 2017), pois a RSC trabalha diretamente com informações sobre suas ações, destarte, a empresa deve fornecer, de forma geral, demonstrativos contábeis que proporcionem informações mais confiáveis, compreensíveis, comparáveis e relevantes quanto ao que foi realizado (Degenhart, Mazzuco, \& Klann, 2017). A informação contábil necessita ser de qualidade, disponibilizando aos usuários informações reais sobre a realidade da empresa (Healy \& Wahlen, 1999). Segundo Ball (2006), situações e práticas distintas podem influenciar a qualidade da informação, possibilitando uma responsabilidade mais assídua e equilíbrio quanto ao que é divulgado, desta forma, refletindo nas demonstrações contábeis divulgadas.

Os debates sobre qualidade das demonstrações contábeis vêm crescendo na literatura, sendo abordados em mercado nacional e internacional. Nesse contexto, são associados de forma geral ao gerenciamento de resultados, relevância e tempestividade da informação (Moura, Theiss, \& Cunha, 2014; Herculano \& Moura, 2015), tratando o uso da informação como algo que pode impactar sobremaneira na empresa. Apresentam-se, ainda, voltados à conduta corporativa (Bandeira, Góis, De Luca, \& Vasconcelos, 2015), onde a má qualidade das demonstrações evidenciadas denota-se reflexiva na tomada de decisão do investidor. Outro fator que pode interferir na qualidade é a existência de uma inconsistência de informações divulgadas, fazendo com que a empresa necessite republicar seus demonstrativos para divulgação de base de referência corrigida (Habib \& Jiang, 2015), desta forma, representando um fator negativo quanto aos dados divulgados em um período anterior.

A republicação de demonstrações contábeis consiste na reapresentação de demonstrativos publicados anteriormente com erros, sejam esses qualitativos ou quantitativos, assim como fraudes organizacionais quanto aos retórios financeiros já divulgados (Benyoussef \& Khan, 2017; Chiudini, Cunha, \& Marques, 2018; He \& Chiang, 2013). Esse instrumento, apresenta-se como um indício da qualidade ruim de informações divulgadas (Akhigbe, Kudla, \& Madura, 2005), podendo impactar na tomada de decisão do investidor (Soares, Motoki, \& Monte-Mor, 2018), e sinalizar situações distintas para o mercado, como manipulação de resultados (Dechow, Ge, \& Schrand, 2010; Marques, Amaral, Souza, Santos, \& Belo, 2017).

Sobre a ótica da Teoria da Sinalização, Spence (1973) expõe que as organizaçoes buscam emitir sinais que sejam determinantes na diminuição da assimetria de informações, possibilitando que os usuários façam suas escolhas com maior clareza, diante das diversas ofertas. A RSC é uma forma de sinalizar ao mercado práticas e preocupações organizacionais que impactam positivamente na visão do investidor (Freitas, Santos, \& Crisóstomo, 2019), desta forma, podendo sinalizar também que apresenta um maior controle e qualidade dos demonstrativos evidenciados (Kim et al., 2012).

Diante da relevância da temática, surgiu o seguinte questionamento: Qual a influência da Responsabilidade Social Corporativa na qualidade das demonstrações contábeis? Com o intuito de responder ao questionamento do estudo, traçou-se como objetivo de pesquisa investigar a influência da RSC na qualidade das demonstrações contábeis de empresas brasileiras, para tal, considerou-se uma amostra de 300 companhias e o interregno de 2015 a 2019.

A qualidade das demonstrações contábeis é fator fundamental para a tomada de decisão (Rezende, 2010; Silva et al., 2017), uma vez que, ao considerar a relevância da informação no poder decisório, qualquer inconsistência nos demonstrativos pode impactar sobremaneira no futuro da organização (Li, Park, \& Wynn, 2018). Nesse contexto, a RSC é uma ferramenta que tem como objetivo trazer benefícios quanto às ações adotadas, mas também quanto a confiança e transparência da informação divulgada (Freitas et al., 2019; Kim et al., 2012).

Deste modo, este estudo justifica-se diante da importância desses parâmetros organizacionais para os stakeholders, uma vez que podem ser determinantes como influenciadores 
do poder decisório (Degenhart et al., 2017), denotando maior clareza para o stakeholder quanto a influência de empresas que tendem a investir mais em RSC e a relevância das informações contábeis (Hedlund, 2021). Nesse ínterim, apresenta a má qualidade da informação medida pela republicação das demonstrações contábeis associada à divulgação de empresas responsáveis socialmente, considerando como medida de baixa qualidade pelo fato de que empresas que republicam seus demonstrativos tendem a não apresentar a informação de forma clara e tempestiva, dificultando a análise dos stakeholders, denotando falhas na divulgação da informação econômico-financeira da entidade (Flanagan, Muse, \& O'Shaughnessy, 2008).

Justifica-se, ainda, a relevância diante da temática para maiores avanços e discursões direcionadas, ao passo que, denota-se como algo pouco exposto na literatura ao tratar da qualidade da informação, principalmente medida pela republicação de demonstrativos contábeis (Soares et al., 2018), nesse sentindo, acredita-se que este estudo contribuirá para ampliar a visão da relação da RSC e a qualidade da informação, considerando o impacto e as consequências desses elementos em empresas brasileiras. Desta feita, o estudo pode revelar contribuições relevantes para investidores, reguladores e diversos stakeholders, como um instrumento de conhecimento efetivo do desenvolvimento e fatores positivos a essas entidades, apontando-se conjuntamente, de grande relevância para maiores esclarecimentos e ampliação da discussão dos temas no âmbito econômico e acadêmico, podendo auxiliar normatizadores e reguladores a compreender mais claramente sobre práticas de negócios das entidades e comportamento de relatórios contábeis republicados à luz da RSC.

\section{FUNDAMENTAÇÃO TEÓRICA}

Nesta seção, são apresentados os posicionamentos teóricos e empíricos que fundamentam o desenvolvimento da linha de raciocínio da pesquisa. Destaca-se ainda nesta seção, a evidenciação do desenvolvimento da hipótese no decorrer das argumentações sobre os construtos do estudo.

\subsection{Responsabilidade social corporativa na qualidade das demonstrações contábeis de empresas brasileiras}

Dentre as práticas organizacionais, a RSC vem tomando grandes proporções, uma vez que, as entidades encontram-se cada vez mais preocupadas no impacto que podem causar ao ambiente em que estão inseridas, apresentando atitudes que minimizem danos (Mcwilliams \& Siegel, 2001), e proporcionem maior transparência (Kim et al., 2012; Baraibar-diez \& Sotorrío, 2018). Deste modo, apresenta benefícios não somente para o ambiente social, mas obtendo efeitos positivos da opinião dos stakeholders diante dessas atitudes (Rao \& Tilt, 2016). De acordo com Servaes e Tamayo (2013), a RSC corresponde ao comprometimento da organização em proporcionar maiores cuidados para o desenvolvimento econômico sustentável, contribuindo assim para avanços superiores na qualidade de vida dos funcionários, famílias, comunidade e sociedade.

No âmbito acadêmico, a RSC vem sendo abordada e medida de diferentes maneiras, dentre elas o Índice de Sustentabilidade Empresarial (ISE) (Nossa et al., 2009), considerado uma ferramenta de análise do desempenho das empresas listadas na B3 ligadas ao aspecto da sustentabilidade corporativa, tomando como base a eficiência econômica, equilíbrio ambiental, justiça social e ainda, governança corporativa (BM\&FBovespa, 2017). Assim como, muitas vezes, associada ao Balanço Social do Modelo IBASE (2017), em que são apresentados benefícios sociais internos, externos e ambientais (Degenhart et al., 2017), e CSRHub que apresenta 12 indicadores de RSC agrupados em quatro categorias, quais sejam: funcionários, meio ambiente, comunidade e governança (Prudêncio, Forte, De Luca, \& Vasconcelos, 2019).

Segundo Scholtens e Kang (2013), entidades que tenham maior preocupação com suas ações sociais e práticas de RSC têm maior probabilidade de sinalizar que são "boas empresas", 
assim como, reflexo positivo quanto às suas atitudes no mercado de capitais (Blacconiere \& Patten, 1994), fortalecendo também sua imagem para os stakeholders (Servaes \& Tamayo, 2013). Nesse contexto, a apresentação de tais características pode refletir na forma como a organização apresenta seus dados, denotando maiores cuidados na elaboração e divulgação de informações econômico-financeiras da empresa.

A informação é vista como um elemento indispensável para o poder decisório, proporcionando maior embasamento para a formação de uma opinião com segurança e refletindo nas próprias ações da empresa (Rezende, 2010). Deste modo, têm-se os relatórios contábeis como representantes definitivos da entidade, evidenciando sua situação patrimonial, financeira, e atuando como fontes indispensáveis sobre o posicionamento e andamento da entidade (Moura et al., 2017).

Nessa perspectiva, a qualidade da informação evidenciada é algo de grande valor, necessitando de maiores cuidados e clareza quanto ao que é divulgado, principalmente por ser elemento essencial para a tomada de decisão do investidor (Chiudini et al., 2018). Ademais, a qualidade das demonstrações contábeis pode trazer inúmeros benefícios, não somente quanto a decisões mais coerentes e concisas (Sousa, Fernandes, Bezerra, \& Ribeiro, 2016), como também para a imagem da empresa no mercado em que está inserida (Bandeira et al., 2015). Lee e Masulis (2009) explanam que a má qualidade gera reflexos nos investidores externos da empresa, ligados a incertezas sobre a condição financeira real da organização, assim como pode induzir uma diminuição de demanda por novos patrimônios, gerando maiores custos e o risco.

A literatura nacional expõe a má qualidade das demonstrações contábeis medida principalmente pelo gerenciamento de resultados (Moura et al., 2014; Herculano \& Moura, 2015), ou má conduta corporativa (Bandeira et al., 2015) ligada à reputação da entidade. Entretanto, a qualidade ruim dos relatórios financeiros pode ser associada a outros fatores, como a republicação das demonstrações contábeis (Li et al., 2018), uma vez que denota a imprecisão material da informação divulgada, apresentando dados que não representam a situação real da empresa, fazendo com que necessite que exista a divulgação dos elementos corrigidos em um momento posterior (Cianci, Clor-proell, \& Kaplan, 2019). A republicação das demonstrações contábeis pode ocorrer de forma voluntária, em que não existe a exigência do órgão regulador, ou compulsória, onde a CVM exige que a informação seja corrigida e reapresentada (Marques et al., 2017). Destarte, a maneira como a entidade é gerenciada pode dizer muito quanto à qualidade da informação divulgada, podendo emitir sinais para as partes interessadas de que a entidade denote maiores controles ou não sobre as demonstrações contábeis apresentadas.

Ao considerar fatores associados como RSC, poucos estudos examinaram a relação de RSC e a qualidade da informação divulgada. Dentre os estudos presentes na literatura, Degenhart et al. (2017) analisaram a relação entre a RSC e a relevância das informações contábeis de empresas brasileiras no período 2011 a 2015. Os autores constataram que a RSC relaciona-se de forma positiva com a relevância das informações contábeis. Kim et al. (2012) examinaram se empresas americanas socialmente responsáveis se comportam de maneira diferente de outras empresas em seus relatórios financeiros quanto à qualidade evidenciada. Concluindo que as empresas socialmente responsáveis têm menos probabilidade de gerenciar os ganhos, manipular atividades e de ser objeto de investigações da SEC.

Nesse ínterim, a emissão de indícios de melhorias organizacionais, como as de empresas que atuam com práticas de RSC, pode sinalizar aspectos confirmatórios ao mercado referentes à qualidade das demonstrações contábeis evidenciadas (Costa, Correia, Paulo, \& Lucena, 2018), possibilitando associar maiores práticas de ações sociais e ambientais como fator que reflete controle, tempestividade e fidedignidade do que é apresentado para os stakeholders, assim sinaliza maiores níveis de qualidade na evidenciação de seus demonstrativos contábeis (Degenhart et al., 2017), destarte, não necessitando republicá-los. Desta feita, desenvolve-se a hipótese do estudo: 
Hipótese geral: Empresas que apresentam práticas de Responsabilidade Social Corporativa divulgam demonstrações contábeis de maior qualidade.

\section{METODOLOGIA}

\subsection{População e amostra}

A população é composta por todas as empresas ativas listadas na B3 S.A. e que estavam presentes no banco de dados Economatica ${ }^{\circledR}$ em 15/02/2020. Para a composição da amostra, foram consideradas as companhias com dados disponíveis para a construção das variáveis, compreendendo o interregno de 2015, 2016, 2017, 2018 e 2019, correspondendo a um espaço temporal de relevante fator comparativo das organizações e para uso de dados em painel (Wooldridge, 2007). Destarte, resultando em uma amostra de 300 empresas.

$\mathrm{Na}$ Tabela 1 apresenta-se a lista de segmento organizacional com base na amostra e considerando o número de entidades associadas.

Tabela 1

Lista dos segmentos das empresas

\begin{tabular}{c|c}
\hline Segmento organizacional & Número de empresas \\
\hline Bens industriais & 51 \\
\hline Comunicações & 5 \\
\hline Consumo cíclico & 72 \\
\hline Consumo não cíclico & 21 \\
\hline Financeiro & 50 \\
\hline Materiais básicos & 28 \\
\hline Petróleo gás e biocombustíveis & 10 \\
\hline Saúde & 17 \\
\hline Tecnologia da informação & 6 \\
\hline Utilidade pública & 40 \\
\hline Total de empresas & $\mathbf{3 0 0}$ \\
\hline
\end{tabular}

Fonte: Elaborado pelos autores.

A partir da Tabela 1 infere-se que 71 empresas da amostra compõem o setor de Consumo cíclico, seguido pelo setor de Bens industriais (51 empresas) e Financeiro (50 empresas), ao passo que o setor de Comunicações (5 empresas), Tecnologia da informação (6 empresas) e Petróleo, gás e biocombustíveis (10 empresas) foram os que apresentaram menor quantidade das empresas da amostra.

\subsection{Variáveis de pesquisa e Coleta de dados}

Para a coleta dos dados, foram utilizados o website da CVM para informações sobre a republicação das demonstrações contábeis das empresas, a Base de dados CSRHub para as práticas de RSC, e Economatica para as informações financeiras.

Na Tabela 2 observam-se a descrição, as fontes e o suporte teórico das variáveis utilizadas na pesquisa. 
Tabela 2

Variáveis utilizadas na pesquisa

\begin{tabular}{|c|c|c|c|c|}
\hline Variável & Proxy & Descrição & Fonte de dados & $\begin{array}{l}\text { Suporte } \\
\text { teórico }\end{array}$ \\
\hline \multicolumn{5}{|c|}{ Variável Dependente } \\
\hline $\begin{array}{c}\text { Baixa qualidade das } \\
\text { demonstrações } \\
\text { contábeis }\end{array}$ & $\begin{array}{l}\text { Republicação } \\
\text { (REPUB) }\end{array}$ & $\begin{array}{c}\text { Variável dummy que } \\
\text { assume valor } 1 \\
\text { quando houver } \\
\text { republicação da } \\
\text { demonstração e valor } \\
0 \text { (zero) caso } \\
\text { contrário }\end{array}$ & Website da CVM & $\begin{array}{c}\text { Chiudini et al. } \\
\text { (2018); Zhang, } \\
\text { Huang e Habid } \\
\text { (2018). }\end{array}$ \\
\hline \multicolumn{5}{|c|}{ Variável Independente } \\
\hline $\begin{array}{c}\text { Responsabilidade Social } \\
\text { Corporativa }\end{array}$ & $\mathrm{RSC}$ & $\begin{array}{l}\text { Métrica geral } \\
\text { CSRHub }\end{array}$ & $\begin{array}{l}\text { Base de dados } \\
\text { CSRHub }\end{array}$ & $\begin{array}{c}\text { Yasser, Al } \\
\text { Mamun, } \\
\text { Ahmed (2017). }\end{array}$ \\
\hline \multicolumn{5}{|c|}{ Variáveis de Controle } \\
\hline Tamanho da empresa & TAM & $\begin{array}{l}\text { Logaritmo natural } \\
\text { ativo total }\end{array}$ & Economatica & $\begin{array}{c}\text { Cao, Du e } \\
\text { Hansen (2017); } \\
\text { Zhang et al. } \\
\text { (2018). }\end{array}$ \\
\hline Endividamento & END & $\begin{array}{c}\text { Relação entre Total } \\
\text { de Passivo e Ativo } \\
\text { Total } \\
\end{array}$ & Economatica $^{\circledR}$ & Tavares (2016). \\
\hline Desempenho & ROA & $\begin{array}{l}\text { Relação entre o } \\
\text { Lucro líquido e o } \\
\text { ativo total } \\
\end{array}$ & Economatica $^{\circledR}$ & $\begin{array}{l}\text { Shahzad et al. } \\
\text { (2020). }\end{array}$ \\
\hline Alavancagem & GAF & Lucro líquido/Ebitda & Economatica $^{\circledR}$ & $\begin{array}{l}\text { Muñoz et al. } \\
(2020) \text {. }\end{array}$ \\
\hline ANO & Dumies ANO & $\begin{array}{l}\text { Assume } 1 \text { para o ano } \\
\text { e } 0 \text { para os demais }\end{array}$ & Economatica $^{\circledR}$ & $\begin{array}{c}\text { Ximenes, e } \\
\text { Ferreira (2021). }\end{array}$ \\
\hline
\end{tabular}

Fonte: Elaborado pelos autores com base na literatura.

A variável dependente do estudo corresponde à Baixa qualidade das Demonstrações Contábeis, medida por meio da republicação dos demonstrativos, analisada por uma variável dummy que assume o valor " 1 " quando houver republicação das demonstrações contábeis e o valor "0" (zero) caso contrário (Zhang et al., 2018), deste modo, considerou-se todos os tipos de republicação (compulsória e voluntária). Como variável independente tem-se a RSC em que considerou-se a métrica de avaliação geral CSRHub (Yasser, Al mamun \& Ahmed, 2017).

A base CSRHub disponibiliza dados de suas dimensões a partir de quatorze indicadores agrupados em quatro categorias: funcionários (indicadores compensação, diversidade e direitos trabalhistas, treinamento, saúde e segurança), meio ambiente (indicadores energia e mudanças climáticas, política ambiental e relatórios, gestão de recursos), comunidade (indicadores desenvolvimento comunitário e filantropia, produtos, direitos humanos e cadeia de suprimentos) e governança (indicadores conselho, ética de liderança, transparência e relatórios), abrangendo, assim, aspectos sociais, ambientais e econômicos (Ribeiro et al., 2017). A média das quatro categorias descritas forma a métrica geral de RSC, desta forma são padronizadas e recebem uma pontuação que varia de $0-100$.

As variáveis de controle são representadas pelo tamanho da empresa, caracterizado pelo logaritmo natural do ativo total (Cao et al., 2017), o endividamento, mensurado pela relação entre total de passivo e ativo total (Tavares, 2016), desempenho mensurado pela relação entre o lucro líquido e o ativo total (Shahzad et al., 2020); Alavancagem, obtida por meio da relação entre o lucro líquido e o Ebitda (Muñoz et al., 2020); e Dumies de ANO. 


\subsection{Procedimentos de Análise dos dados}

Com o propósito de atender ao objetivo do estudo e testar a hipótese formulada, inicialmente realizou-se uma análise descritiva para melhor conhecimento das variáveis, considerando as variáveis contínuas do estudo. Ademais, foi feito o teste de diferença entre médias da RSC e republicação das demonstrações contábeis, para tal, considerou-se dois grupos: G1 de empresas que não republicaram e G2 de empresas que republicaram suas informações financeiras. Como forma complementar realizou-se o teste de correlação de Pearson entre as variáveis e a republicação das demonstrações contábeis. Por fim, foi feita a análise de regressão logística com dados em painel, para se inferir acerca da influência da RSC na qualidade das demonstrações contábeis divulgadas.

A partir da análise de dados em painel, a organização dos dados ocorre em séries temporais que mostram a evolução das variáveis não unicamente em um determinando instante de tempo, mas ao longo do tempo e diante das observações realizadas (Fávero et al., 2009).

Para a análise de dados em painel foram observadas as abordagens de pooled independent cross-sections (POLS - pooled ordinary least squares), efeitos fixos (fixed effects) e efeitos aleatórios (random effects). No caso do modelo pooled não se verifica elementos ligados às diferenças comportamentais das entidades dentro do conjunto de dados observados, entretanto, a abordagem de efeitos fixos leva em consideração a existência de diferenças significativas no comportamento das empresas. O modelo de efeitos aleatórios analisa as diferenças de cada indivíduo como aleatória, em que se verifica semelhanças com as observações da amostra (Fávero et al., 2009).

Dessa forma, a Equação abaixo apresenta o modelo econométrico do estudo.

$$
R E P U B i j=\beta 0+\beta 1 R S C i j+\beta 2 T A M i j+\beta 3 E N D i j+\beta 4 R O A i j+\beta 5 G A F i j+\varepsilon_{i j}
$$

Ademais, destaca-se que foram realizados também o teste Chow para identificar o modelo (Pool ou efeitos fixos). Em seguida, o teste de LM (Lagrange multiplier) de Breusch-Pagan (para Pool e efeitos aleatórios) e, por último, o Teste de Hausman, para identificar o mais representativo (fixo ou aleatório) (Fávero et al., 2009).

Ademais, antes da análise de regressão, foi realizada uma estatística descritiva das variáveis de interesse do estudo, do qual foram extraídas medidas de tendência central e variabilidade, com o intuito de verificar uma prévia dos comportamentos dos dados. Assim como, foi realizado o teste de diferença entre médias (teste t de student) para a comparação das empresas que não republicam e republicam suas demonstrações contábeis, mas também utilizou-se da correlação de Pearson para verificar a associação entre as variáveis do estudo, tendo em vista que os dados atenderam ao pressuposto de normalidade.

\section{APRESENTAÇÃO E ANÁLISE DOS RESULTADOS}

\subsection{Estatística descritiva}

Com a finalidade de se compreender a distribuição dos dados da pesquisa, apresentam-se as características da amostra das empresas analisadas nesse estudo. Inicialmente, apresenta-se um panorama das variáveis do estudo, denotando de forma geral sua distribuição, em seguida verificase diferenças, relação e influência entre as variáveis.

A Tabela 3 apresenta a descrição das variáveis contínuas e discretas utilizadas na pesquisa. 
Tabela 3

Caracterização das variáveis contínuas e discretas

\begin{tabular}{c|c|c|c|c}
\hline Variáveis & Mínimo & Máximo & Média & Desvio padrão \\
\hline RSC & 34,0 & 67,0 & 53,87 & 6,097 \\
TAM & 2,0365 & 21,276 & 14,61 & 2,526 \\
END & 0 & 46,75 & 0,922 & 2,647 \\
ROA & $-13,30$ & 2,217 & 0,174 & 3,653 \\
GAF & $-140,974$ & 282,839 & 1,004 & 11,71 \\
\hline
\end{tabular}

Fonte: Dados da pesquisa.

Pela Tabela 3 observa-se que dentre as empresas que apresentam ações de RSC, denota-se que sinalizaram um valor médio de 53,87, sendo que há empresa que apresentou um mínimo de 34 e um máximo de 67, sendo que seu valor é bastante heterogêneo entre as empresas (Desvio padrão = 6,097). As demais variáveis (TAM, END, ROA, GAF) mostraram-se dispersas, evidenciados valores heterogêneos entre as empresas em relação aos seus valores.

Reflexo disso, diversos estudos destacam que empresas brasileiras vêm sinalizando republicação de seus demonstrativos (Tavares, 2016; Chiudini et al., 2018; Soares et al., 2018).

No cenário brasileiro, embora a maioria das republicações sejam voluntárias, podendo em um primeiro instante sinalizar maior transparência para o mercado, Palmrose, Richardson e Scholz (2004), Cao, Myers e Omer (2012) e Marque et al. (2017) destacam que isso pode apresentar efeitos adversos relevantes sobre o custo de capital e reputação da empresa. Assim, a qualidade da informação divulgada pode ser afetada por inúmeros fatores contextuais, inclusive, pela RSC (Degenhart et al., 2017).

No que tange à RSC, verifica-se que as empresas brasileiras estão cada vez mais socialmente responsáveis, tendo em vista que há maior atenção em relação às suas condutas pela sociedade, evidenciando que há uma preocupação para além dos ganhos econômicos. Assim, como ponderado por Albuquerque Filho et al. (2019) página 13 apud Beltratti (2005) "as empresas socialmente responsáveis são muitas vezes também as empresas mais respeitadas e lucrativas".

\subsection{Teste de médias}

Na Tabela 4 verifica-se o teste de médias da RSC entre as empresas que não republicam e que republicam suas demonstrações contábeis, denotando as semelhanças e diferenças entre as variáveis RSC e republicação.

Tabela 4

Teste de médias da RSC entre as empresas que republicam e não republicam suas demonstrações contábeis

\begin{tabular}{c|c|c|c|c|c|c}
\hline \multirow{2}{*}{ Variável } & \multirow{2}{*}{ REPUB } & \multirow{2}{*}{$\mathbf{N}$} & \multicolumn{2}{|c|}{ Teste t de Student } & \multicolumn{2}{c}{ Teste de Levene } \\
\cline { 4 - 7 } & & & Média & Sig. & F & Sig. \\
\hline \multirow{2}{*}{ RSC } & G1 & 170 & 54,010 & 0,407 & \multirow{2}{*}{0,001} & 0,981 \\
\hline
\end{tabular}

Fonte: Elaborada pelos autores

*significante a $1 \%$.

Legenda: G1- empresas que não republicaram e G2- empresas que republicaram.

Pela Tabela 4 infere-se que há diferenças estatisticamente significantes na RSC nas empresas que republicam suas demonstrações contábeis comparativamente às empresas que não republicam suas demonstrações contábeis. A RSC das empresas que não republicam (G1) é maior do que nas empresas que republicam (G2), sinalizando que as empresas que não republicam suas demonstrações contábeis tendem a apresentar maior RSC. 
Nesse contexto, tem-se a RSC como um instrumento que auxilia no aumento de benefícios quanto às ações adotadas, mas também quanto à confiança e transparência da informação divulgada (Freitas et al., 2019; Kim et al., 2012), neste caso, a não republicação de informações contábeis. Ao passo que, segundo Helou Neto e Pereira (2010), as republicações no cenário brasileiro evidenciam uma reação negativa quanto ao mercado quando direcionada a uma republicação exigida pela CVM.

\subsection{Correlação de Pearson}

A Tabela 5 demonstra a correlação de Pearson entre as variáveis e a REPUB.

Tabela 5

\section{Correlação das variáveis}

\begin{tabular}{c|c|c}
\hline Variáveis & Resultado & Resultado Observado \\
\cline { 3 - 3 } & Esperado & $\boldsymbol{R} \boldsymbol{E P U \boldsymbol { B }}$ \\
\hline RSC & - & $-0,39(* *)$ \\
TAM & - & $-0,08(* *)$ \\
END & + & $+0,11(\mathrm{NS})$ \\
ROA & - & $+0,02(\mathrm{NS})$ \\
GAF & - & $+0,03(\mathrm{NS})$ \\
\hline
\end{tabular}

Fonte: Elaborada pelos autores

** significante a 5\%; NS: Não significante.

Pela Tabela 5 depreende-se que há uma correlação inversa entre a RSC e a Republicação das Demonstrações Contábeis (REPUB). Além disso, nota-se que o tamanho das empresas também apresentou uma associação negativa com a REPUB. As variáveis END, ROA e AF não apresentaram significância estatística. Dessa forma, destaca-se que quanto maior a RSC e o tamanho, menor a republicação das demonstrações contábeis pelas empresas.

Tais resultados corroboram com o que afirma Bischoff, Finley e Leblanc (2008), de que no mercado acionário a notícia da republicação denota algo negativo, sinalizando a possibilidade de fraude ou ausência de demonstrações com qualidade. Assim, levando em consideração a qualidade da informação, a relação negativa entre a RSC e a REPUB vai de encontro aos achados de Schuster e Klann (2019) e Malo-Alain, Melegy e Ghoneim (2019), que também analisaram os efeitos da RSC na qualidade da informação contábil.

Portanto, infere-se que na medida que as empresas expõem suas ações de RSC como forma de transparência, também tendem a apresentar estímulos para potencializar a qualidade de suas informações, mediante a não republicação de seus demonstrativos.

\subsection{Regressão logística}

A Tabela 6 evidencia a influência da RSC na REPUB.

Tabela 6

Regressão logística da influência da RSC na REPUB

\begin{tabular}{c|c|c|c}
\hline \multirow{2}{*}{ Variável } & \multicolumn{2}{c}{ Republicação das demonstrações Financeiras } \\
\cline { 2 - 4 } & \multicolumn{2}{|c}{ Colinearidade } \\
\hline \multicolumn{2}{c|}{ Efeitos aleatórios I } & Tolerância & VIF \\
\hline RSC & $-0,016^{* *}$ & 0,976 & 1,025 \\
TAM & $-0,083^{* * *}$ & 0,907 & 1,103 \\
END & 0,062 & 0,833 & 1,200 \\
ROA & 0,032 & 0,899 & 1,112 \\
GAF & $-0,005$ & 0,991 & 1,009 \\
Constante & 2,627 & - & - \\
Dammy_ANO & Sim & - & - \\
\hline
\end{tabular}




\begin{tabular}{c|c} 
Wald Chi2 & 5,176 \\
p-value & $0,000^{*}$ \\
$\mathrm{R}^{2}$ & 0,150 \\
VIF médio & 1,089
\end{tabular}

Fonte: Elaborada pelos autores

$(*),(* *),(* * *)$ significante a $1 \%, 5 \%$ e $10 \%$, respectivamente.

Na Tabela 6 observa-se que o Wald Chi2 foi significante a 1\%, possibilitando a análise do modelo. O poder explicativo do modelo $\left(\mathrm{R}^{2}\right)$ corresponde a $15 \%$ e o VIF sinaliza a não multicolinearidade entre as variáveis independentes (VIF<5).

Pela regressão, verifica-se que a RSC apresentou significância estatística a 5\%. O coeficiente negativo ( $\mathrm{RSC}=-0,016$ ) evidencia uma influência negativa da RSC na REPUB. Assim, denota-se que quanto maior a RSC, menor a probabilidade de Republicação das Demonstrações Contábeis (REPUB) pelas empresas, com impactos subsequentes na qualidade de suas informações contábeis. Dessa maneira, aceita-se a hipótese deste estudo de que empresas que apresentam práticas de Responsabilidade Social Corporativa divulgam demonstrações contábeis de maior qualidade.

Nesse ínterim, percebe-se que a emissão de indícios de melhorias organizacionais pode sinalizar aspectos confirmatórios ao mercado quanto à qualidade das demonstrações contábeis evidenciadas (Costa et al., 2018), apresentando a RSC como ferramenta positiva com a relevância das informações contábeis (Degenhart et al., 2017), corroborando com os estudos de Kim et al. (2012) e Scholtens e Kang (2013) de que entidades que tenham maior preocupação com suas ações sociais e práticas de RSC, tem maior probabilidade de sinalizar que são "boas empresas" com reflexo em suas informações evidenciadas.

Além disso, como ponderado por Kim et al. (2012), na medida que a RSC é uma questão de interesse ascendente, tendo em vista que os investidores, clientes e partes interessadas exigem uma maior transparência, as empresas conforme corroboradas pela Teoria da sinalização, precisam divulgar suas informações para o mercado, podendo inserir nesse contexto suas ações ambientais, sociais e econômicas.

Nesse cenário, a relação negativa entre a RSC e a REPUB corrobora com o evidenciado por Albuquerque Filho et al. (2019), que destacaram que a RSC induz maior transparência, podendo contribuir com a melhoria da marca e reputação da empresa aliviando suas preocupações sobre consultas negativas, como por exemplo, republicar suas demonstrações contábeis.

Ademais, verificou-se também que o tamanho da empresa foi negativo e estatisticamente significante $(\mathrm{TAM}=-0,083, \mathrm{p}$-value $<1 \%)$, evidenciando que maiores empresas tendem a apresentar menor probabilidade de republicação e, consequentemente, tendem a apresentar melhor qualidade de suas informações. Outrossim, as demais variáveis (END, ROA e AF) não apresentaram significância estatística.

Destaca-se que, embora tenha-se verificado em estudos prévios uma relação positiva do tamanho na republicação (Khalil \& Ozkan, 2016; Marques et al., 2017), é relevante destacar que no contexto brasileiro, a maioria das republicações ocorrem de maneira espontânea (voluntária) (Marques et al., 2017). Isso significa, de acordo com Marques (2016), que não necessariamente tratam-se de republicações ocasionadas por algum tipo de irregularidade. O estudo de Helou Neto e Pereira (2010), por exemplo, evidenciou que, no cenário brasileiro, apenas as republicações obrigatórias geram um efeito negativo nas informações divulgadas pelas empresas.

\section{CONCLUSÕES FINAIS}

Com o propósito de investigar a influência da RSC na qualidade das demonstrações contábeis de empresas brasileiras, realizou-se uma revisão da literatura com base na temática, 
estudos similares e uma análise dos dados por meio de tratamento estatístico. Nesse contexto, considerou-se a variável Republicação das Demonstrações Contábeis como fator de baixa qualidade da informação e a métrica geral CSRHub como medida de RSC.

A partir dos resultados do estudo, constatou-se que existem diferenças estatisticamente significantes na RSC nas empresas que republicam suas demonstrações contábeis comparativamente às empresas que não republicam seus demonstrativos, denotando que as empresas que não republicam seus demonstrativos tendem a apresentar uma maior RSC, destarte, uma maior preocupação das empresas que possuem maiores investimentos em RSC com a divulgação de informações de qualidade.

Tal afirmação pode ser constatada ao longo das outras análises estatísticas realizadas, como na análise de correlação, que apresentou uma correlação negativa entre a RSC e a republicação das demonstrações contábeis, destarte, apontando-se que quanto maior a RSC e o tamanho organizacional, menor a republicação das demonstrações contábeis pelas empresas.

Ao realizar a análise de regressão em busca de verificar a influência entre as variáveis, constatou-se ainda uma influência negativa da RSC na republicação das demonstrações contábeis, verificando que quanto maior a RSC, menor a probabilidade de republicação das demonstrações contábeis pelas empresas.

Nesse ínterim, empresas com maiores níveis de RSC apresentam maiores cuidados com a informação divulgada, assim, uma possibilidade maior de não republicarem suas informações contábeis, ou seja, maior qualidade da informação denotada. Dessa maneira, aceita-se a hipótese deste estudo de que empresas que apresentam RSC (com práticas voltadas para funcionários, meio ambiente, comunidade e governança, abrangendo, assim, aspectos sociais, ambientais e econômicos) divulgam demonstrações contábeis de maior qualidade.

A partir da análise da influência da RSC na qualidade das demonstrações contábeis de empresas brasileiras, pela perspectiva da republicação de demonstrativos contábeis, foi possível compreender a relação entre a RSC e a qualidade da informação, possibilitando que sejam apresentadas informações sobre aspectos organizacionais que possibilitam ao acionista tomar a melhor decisão, o qual, em geral, procura por entidades que denotem maior confiança e responsabilidade.

Destarte, este estudo contribui para a literatura a partir da identificação dessa lacuna, pois são apontados avanços no conhecimento, debates com base em estudos anteriores e resultados encontrados, em especial sobre a ótica da Teoria da Sinalização no campo das organizações no Brasil, apresentando maiores evidências da relevância das organizações investirem em RSC e, posteriormente, divulgá-la nos relatórios, possibilitando influenciar a qualidade da informação evidenciada, assim como proporcionar benefícios frente às ações sociais e ambientais, denotando a empresa com uma postura mais ética. Salienta-se, ainda, que os resultados aqui explicitados, bem como as considerações apresentadas, denotam subsídios para uma análise de organizações com características e mercado específico, desta forma, limitando-se as empresas brasileiras listadas na B3, não possibilitando auferir informações ligadas a outros mercados.

Para estudos futuros, sugere-se utilizar medidas distintas de RSC ou qualidade da informação; estudar esses preceitos em outros mercados; e analisar a RSC como moderadora em relações como a competitividade e efeitos associados, permitindo, assim, uma visão mais precisa de fatores marcantes e que podem impactar o futuro organizacional.

\section{REFERENCIAS}

Akhigbe, A., Kudla, R. J., \& Madura, J. (2005). Why are some corporate earnings restatements more damaging? Applied financial economics, 15(5), 327-336. 
Albuquerque Filho, A. R., Sousa, A. L. C., Silveira Lopes, H. S., Guimarães, D. B., \& Ponte, V. M. R. (2019). Influência da Internacionalização e da Governança Corporativa na Responsabilidade Social Corporativa. Iberoamerican Journal of Strategic Management, 18(3), 397-419.

Ball, R. (2006). International Financial Reporting Standards (IFRS): pros and cons for investors. Accounting and business research, 36(sup1), 5-27.

Bandeira, M. T. D. S. S., Góis, A. D., Luca, M. M. M., \& Vasconcelos, A. C. (2015). Reputação corporativa negativa e o desempenho empresarial. Revista de Contabilidade e Organizações, 9(24), 71-83.

Baraibar-Diez, E., \& Sotorrío, L. L. (2018). O efeito mediador da transparência na relação entre responsabilidade social corporativa e reputação corporativa. Revista Brasileira de Gestão de Negócios, 20, 05-21.

BenYoussef, N., \& Khan, S. (2017). Identifying fraud using restatement information. Journal of Financial Crime, 24(4), 620-627.

Berens, G., Van Riel, C. B., \& Van Rekom, J. (2007). The CSR-quality trade-off: when can corporate social responsibility and corporate ability compensate each other? Journal of Business Ethics, 74(3), 233-252.

Bischoff, J., Finley, J., \& LeBlanc, D. (2008). Financial statement restatements: Causes and effects. Tennessee CPA Journal, 5-7.

Blacconiere, W. G., \& Patten, D. M. (1994). Environmental disclosures, regulatory costs, and changes in firm value. Journal of accounting and economics, 18(3), 357-377.

Cao, L., Du, Y., \& Hansen, J. Ø. (2017). Foreign institutional investors and dividend policy: Evidence from China. International Business Review, 26(5), 816-827.

Cao, Y., Myers, L. A., \& Omer, T. C. (2012). Does company reputation matter for financial reporting quality? Evidence from restatements. Contemporary Accounting Research, 29(3), 956-990.

Chiudini, V., Cunha, P. R., \& Marques, L. (2018). Relação entre a republicação das demonstrações contábeis e o Audit Delay. Revista Catarinense da Ciência Contábil, 17(51), 89-107.

Cianci, A. M., Clor-Proell, S. M., \& Kaplan, S. E. (2019). How do investors respond to restatements? Repairing trust through managerial reputation and the announcement of corrective actions. Journal of Business Ethics, 158(2), 297-312.

Costa, I. L. D. S., Correia, T. D. S., Paulo, E., \& Lucena, W. G. L. (2018). Impacto do disclosure voluntário: valor da empresa e informações socioambientais nas companhias abertas. Revista Contabilidade, Gestão e Governança-CGG, 21(2), 271-287.

Dechow, P., Ge, W., \& Schrand, C. (2010). Understanding earnings quality: A review of the proxies, their determinants and their consequences. Journal of accounting and economics, 50(23), 344-401. 
Degenhart, L., Mazzuco, M. S. A., \& Klann, R. C. (2017). Relevância das informações contábeis e a responsabilidade social corporativa de empresas brasileiras. Base Revista de Administração e Contabilidade da UNISINOS, 14(3), 157-168.

Fávero, L. P., Belfiore, P., Silva, F. D., \& Chan, B. L. (2009). Análise de dados: modelagem multivariada para tomada de decisões. Rio de Janeiro: Elsevier.

Flanagan, D. J., Muse, L. A., \& O'Shaughnessy, K. C. (2008). An overview of accounting restatement activity in the United States. International Journal of Commerce and Management, 18(4), 363-381.

Freitas, M. R. O., Santos, S. M., \& Crisóstomo, V. L. (2019). Nível de abrangência da informação ambiental divulgada nos relatórios de sustentabilidade de empresas brasileiras com potencial de impacto ao meio ambiente. Revista Contabilidade e Controladoria, 10(3), 143-161.

Habib, A., \& Jiang, H. (2015). Corporate governance and financial reporting quality in China: A survey of recent evidence. Journal of International Accounting, Auditing and Taxation, 24, 2945 .

He, L. J., \& Chiang, H. T. (2013). Market reaction to financial statement restatement: A study on the information and insurance role of auditors. Advances in Management and Applied Economics, 3(4), 37.

Healy, P. M., \& Wahlen, J. M. (1999). A review of the earnings management literature and its implications for standard setting. Accounting horizons, 13(4), 365-383.

Hedlund, P. R. (2021). Influência da responsabilidade social corporativa na qualidade das informações contábeis reportadas ao usuário. Dissertação de Mestrado, Universidade Regional do Noroeste do Estado do Rio Grande do Sul.

Helou Neto, F. H., \& Pereira, C. C. (2010). Impacto da republicação de demonstrações financeiras no preço das ações de empresas brasileiras. Revista Contemporânea de Contabilidade, 7(14), 29-50.

Herculano, H. D. A., \& Moura, G. D. (2015). Informação contábil e concentração acionária: Análise sob a ótica da persistência e da oportunidade. Revista Ambiente Contábil, 7(2), 231247.

Khalil, M., \& Ozkan, A. (2016). Board independence, audit quality and earnings management: Evidence from Egypt. Journal of Emerging Market Finance, 15(1), 84-118.

Kim, Y., Park, M. S., \& Wier, B. (2012). Is earnings quality associated with corporate social responsibility? The accounting review, 87(3), 761-796.

Koprowski, S., Krein, V., Barichello, R., Mazzioni, S., \& Dal Magro, C. B. (2019). Influência das conexões políticas e da evidenciação socioambiental no custo de capital. Contextus-Revista Contemporânea de Economia e Gestão, 17(2), 98-128. 
Lee, G., \& Masulis, R. W. (2009). Seasoned equity offerings: Quality of accounting information and expected flotation costs. Journal of Financial Economics, 92(3), 443-469.

Li, Y., Park, Y. I., \& Wynn, J. (2018). Investor reactions to restatements conditional on disclosure of internal control weaknesses. Journal of Applied Accounting Research, 19(3), 423-439.

Malo-Alain, A. M., Melegy, M. M. A. H., \& Ghoneim, M. R. Y. (2019). The effects of sustainability disclosure on the quality of financial reports in Saudi business environment. Academy of Accounting and Financial Studies Journal, 23(5), 1-12.

Marques, V. A., Amaral, H. F., Souza, A. A., Santos, K. L., \& Belo, P. H. R. (2017). Determinantes das republicações no mercado brasileiro: Uma análise a partir dos incentivos ao gerenciamento de resultados. Revista de Educação e Pesquisa em Contabilidade (REPeC), 11(2), 191-213.

Marques, V. (2016). Qualidade das Informações Financeiras e o Ambiente Regulatório: Evidências Empíricas no Período de 1998-2013. Tese de Doutorado em Administração, Centro de Pós-Graduação e Pesquisas em Administração - CEPEAD, Universidade Federal de Minas Gerais. Belo Horizonte, MG, Brasil.

McWilliams, A., \& Siegel, D. (2001). Corporate social responsibility: A theory of the firm perspective. Academy of management review, 26(1), 117-127.

Moura, E. A., Dias, A. C., Machado, A. C. S. C., \& Santos, A. T. R. (2017). Estudo de caso da situação econômico-financeira da empresa Arezzo indústria e comércio SA. Revista de Trabalhos Acadêmicos-Universo, 2(3).

Moura, G. D., Theiss, V., \& Cunha, P. R. (2014). Ativos intangíveis e gerenciamento de resultados: uma análise em empresas brasileiras listadas na BM\&FBovespa. BASE-Revista de Administração e Contabilidade da Unisinos, 11(2), 111-122.

Muñoz, R. M., Pablo, J. D. S. D., Salinero, Y., \& Peña, I. (2020). Risk measures and the riskreturn paradox: an analysis in the context of the economic crisis. Revista Brasileira de Gestão de Negócios, 22, 348-362.

Nossa, V., Cezar, J. F., Silva Junior, A., Baptista, É. C. S., \& Nossa, S. N. (2009). A relação entre o retorno anormal e a responsabilidade social e ambiental: um estudo empírico na bovespa no período de 1999 a 2006. BBR-Brazilian Business Review, 6(2), 121-136.

Palmrose, Z. V., Richardson, V. J., \& Scholz, S. (2004). Determinants of market reactions to restatement announcements. Journal of accounting and economics, 37(1), 59-89.

Prudêncio, P. A., Forte, H. C., De Luca, M. M. M., \& Vasconcelos, A. C. (2019). Disclosure ambiental negativo e desempenho em empresas listadas na B3. Revista de Gestão Social e Ambiental, 13(2), 58-74.

Rao, K., \& Tilt, C. (2016). Board composition and corporate social responsibility: The role of diversity, gender, strategy and decision making. Journal of Business Ethics, 138(2), 327-347. 
Rezende, D. A. Sistema de informações organizacionais: guia prático para projetos em cursos de administração, contabilidade e informática. (4a ed.). São Paulo, 2010.

Ribeiro, F., Alves, T. A., Taffarel, M., \& Menon, G. (2017). Responsabilidade social corporativa e o desempenho financeiro no setor de energia elétrica: um estudo com modelo de dados em painéis. Gestão \& Regionalidade, 33(99), 39-54.

Scholtens, B., \& Kang, F. C. (2013). Corporate social responsibility and earnings management: Evidence from Asian economies. Corporate Social Responsibility and Environmental Management, 20(2), 95-112.

Schuster, H. A., \& Klann, R. C. (2019). Responsabilidade Social Corporativa e Gerenciamento de Resultados por Accruals. Contabilidade Vista \& Revista, 30(1), 01-26.

Servaes, H., \& Tamayo, A. (2013). The impact of corporate social responsibility on firm value: The role of customer awareness. Management science, 59(5), 1045-1061.

Shahzad, F., Fareed, Z., Wang, Z., \& Shah, S. G. M. (2020). Do idiosyncratic risk, market risk, and total risk matter during different firm life cycle stages? Physica A: Statistical Mechanics and its Applications, 537, 122-550.

Silva, G. C., Takamatsu, R. T., \& Avelino, B. C. (2017). Adesão aos Níveis Diferenciados de Governança Corporativa e Qualidade das Informações Contábeis. ConTexto, 17(35), 89-104.

Silva, J. P. D., Noriller, R. M., Silva, C. A. T., \& Niyama, J. K. (2018). Earning quality e o desempenho das companhias brasileiras de capital aberto. Revista Ibero-Americana de Estratégia, 17(2), 108-124.

Soares, C. R., Motoki, F. Y. S., \& Monte-Mor, D. S. (2018). IFRS e probabilidade de republicação: Um estudo das empresas brasileiras listadas na Bovespa. Revista Catarinense da Ciência Contábil, 17(52).

Sousa, M. A. B., Fernandes, C. F., Bezerra, R. P. P., \& Ribeiro, S. P. (2016). Qualidade da informação contábil: uma análise de suas características com base na percepção do usuário externo. Iberoamerican Journal of Industrial Engineering, 8(15), 208-227.

Spence, M. (1978). Job market signaling. In Uncertainty in economics (pp. 281-306). Academic Press, 2081-306.

Tavares, H. L. M. (2016). O endividamento e a qualidade da informação financeira (Master's thesis). Dissertação de Mestrado, Universidade Lusófona de Humanas e Tecnologias.

Wooldridge, J. M. (2007). Inverse probability weighted estimation for general missing data problems. Journal of econometrics, 141(2), 1281-1301.

Ximenes, F. K. A. A., \& Ferreira, F. R. (2021). O efeito das práticas ambientalmente responsáveis sobre a agressividade fiscal: Uma análise das empresas participantes do Índice Carbono Eficiente-ICO2. Pensar Contábil, 22(79), 60-69. 
Yasser, Q. R., Al Mamun, A., \& Ahmed, I. (2017). Corporate social responsibility and gender diversity: Insights from Asia Pacific. Corporate Social Responsibility and Environmental Management, 24(3), 210-221.

Zhang, H., Huang, H. J., \& Habib, A. (2018). The effect of tournament incentives on financial restatements: Evidence from China. The International Journal of Accounting, 53(2), 118-135.

\section{AGRADECIMENTOS}

Os autores agradecem ao Programa Pesquisa Produtividade da ESTÁCIO FIC. 